\title{
EFEITOS DO TREINO DE ENDURANCE E DE FORÇA EM PACIENTES COM DOENÇA PULMONAR OBSTRUTIVA CRÔNICA: UM ESTUDO DE REVISÃO
}

\author{
Alana Oliveira Mangabeira Fisioterapeuta graduada pela Escola \\ Bahiana de Medicina e Saúde Pública. \\ Membro do Grupo de Pesquisa em \\ Fisioterapia Cardiovascular e \\ Respiratória da Bahiana. \\ Luciana Bilitário Macedo \\ Fisioterapeuta, mestre em Medicina e \\ Saúde Humana, docente da Escola \\ Bahiana de Medicina e Saúde Pública e \\ da Universidade do Estado da Bahia. \\ Membro do Grupo de Pesquisa em \\ Fisioterapia Cardiovascular e \\ Respiratória da Bahiana.
}

\begin{abstract}
Resumo
Introdução: A Doença Pulmonar Obstrutiva Crônica (DPOC) é uma enfermidade crônica (causada principalmente pelo tabagismo) que se caracteriza pela obstrução não totalmente reversível do fluxo aéreo. Os pacientes podem apresentar dispnéia, intolerância ao exercício e mudanças na percepção da qualidade de vida. Estas manifestações melhoram discretamente com terapias clínicas, porém, o condicionamento físico realizado a partir de treinamentos para endurance e força parece fundamental visto que otimiza a condição cardiorrespiratória e musculoesquelética, diminui a sintomatologia e aumenta a capacidade para o exercício. Objetivo: Buscar evidências sobre a influência destes treinos direcionados para a musculatura periférica e seu impacto na dispnéia, tolerância ao esforço e na qualidade de vida desta população. Metodologia: Trata-se de uma revisão de atualização de tema. Utilizaram-se livros e artigos (publicados entre 2000 e 2011) nas bibliotecas virtuais Lilacs; Scielo; Bireme; PubMed; e MedLine coletados entre setembro a novembro de 2011. Resultados: Foram encontrados seis artigos (publicados entre 2001 a 2010) que demonstraram benefícios dos treinos com freqüência mínima de 2 a 3 vezes/semana durante um período mínimo de seis semanas, sobre as variáveis, principalmente no estadiamento moderado e grave da DPOC. Conclusão: O tratamento para pacientes com DPOC deve englobar, além do tratamento conservador, treinos aeróbicos e anaeróbicos dos músculos periféricos, sendo necessários estudos cujos protocolos sejam definidos por volume e intensidade de treinamento.
\end{abstract}

Palavras-chave: Doença pulmonar obstrutiva crônica; Força muscular; Dispnéia.

\section{EFFECTS OF ENDURANCE AND STRENGTH TRAINING IN PATIENTS WITH CHRONIC OBSTRUCTIVE PULMONARY DISEASE}

\begin{abstract}
Introduction: Chronic Obstructive Pulmonary Disease (COPD) is a chronic disease (mainly caused by smoking) that is characterized by a non-reversible airflow limitation. Patients may present with dyspnea, exercise intolerance and changes in the perception of quality of life. These manifestations with clinical therapies improve slightly, but the fitness achieved from training for endurance and strength seems essential since optimizes cardiorespiratory and musculoskeletal condition, reduces symptoms and improves exercise capacity. Objective: To find evidence about the influence of the execise training designed to the peripheral muscles and its impact on dyspnea, exercise tolerance and quality of life. Methods: This is a revision update theme. We used books and articles (published between 2000 and 2011) in virtual libraries Lilacs, SciELO; Bireme; PubMed, MedLine and collected between September and November 2011. Results: Six articles (published from 2001 to 2010), which demonstrated benefits of training with a minimum frequency of 2 to 3 times / week for at least six weeks on the variables were found, especially in staging moderate and severe COPD. Conclusion: The treatment for patients with COPD should include, in addition to conservative treatment, aerobic and anaerobic training of peripheral muscles, and standartized studies containing volume and intensity training are needed.
\end{abstract}

Keywords: Chronic obstructive pulmonary disease; Muscle strength; Dyspnea. 


\section{INTRODUÇÃO}

A Doença Pulmonar Obstrutiva Crônica (DPOC) é definida como uma enfermidade respiratória que se caracteriza pela presença de obstrução crônica do fluxo aéreo e não é totalmente reversível. É geralmente progressiva e está associada a uma resposta anormal dos pulmões diante da constante inalação de partículas ou gases tóxicos, causada principalmente pelo hábito de fumar, além de relacionar-se com infecções respiratórias graves na infância, desnutrição, fatores socioeconômicos, prematuridade e deficiência genética de alfa-1 antitripsina. ${ }^{(1,2)}$

No Brasil estima-se que em adultos maiores de 40 anos exista uma prevalência de $12 \%$ da população com DPOC e que o número de óbito por esta patologia tenha aumentando nos últimos 20 anos, o que a engloba hoje no grupo das oito doenças que mais matam no mundo. ${ }^{(1,3)}$

Os principais sintomas manifestados por indivíduos acometidos pela obstrução crônica são tosse com expectoração, dispnéia progressiva e intolerância ao exercício. Esta intolerância pode ser atribuída tanto a limitação ventilatória quanto à disfunção muscular esquelética desenvolvida por descondicionamento crônico, sendo a redução da massa muscular um fator de previsão independente para a mortalidade nestes pacientes. ${ }^{(2)}$

Os treinamentos de endurance (aeróbico) e força (anaeróbico) são adequados para o aumento da resistência e força muscular, respectivamente. $O$ treino aeróbico consiste na realização de exercícios em esteiras e bicicletas com baixa intensidade e longo tempo e o treino de força inclui exercícios com peso/ carga elevadas e baixas repetições de movimento. ${ }^{(4)}$

Evidências recentes mostram que o treinamento da musculatura periférica é a estratégia adequada para atingir-se fortalecimento de membros superiores e inferiores, aumento na capacidade submáxima de exercício e redução da dispnéia. Tendo em vista que a função ventilatória dos portadores de DPOC pode ser melhorada apenas discretamente por terapias clínicas, o condicionamento físico tem papel fundamental com a finalidade de reduzir a demanda respiratória e a sensação de dispnéia. ${ }^{(1)}$

Desta forma, é evidente a importância de realizar-se uma busca e reunir evidências que discorram sobre a influência do treinamento de endurance e força da musculatura periférica na dispnéia, tolerância ao esforço e na qualidade de vida de indivíduos com DPOC. Sendo assim, o objetivo deste estudo foi buscar evidências sobre a influência destes treinos, direcionados 
para a musculatura periférica, na dispnéia, tolerância ao esforço e na qualidade de vida desta população.

\section{METODOLOGIA}

O presente estudo consiste em uma revisão narrativa para atualização de tema. A busca pelos materiais foi feita em livros e em artigos publicados entre os anos de 2000 até 2011, existentes nas bases de dados virtuais Bireme, Lilacs e Pubmed. A coleta foi realizada entre setembro a novembro de 2011, utilizando-se das seguintes palavras-chaves: fisioterapia na DPOC (therapy in COPD), treinamento muscular na DPOC (muscle training in COPD), dispnéia na DPOC (dyspnea in COPD).

Os critérios de inclusão definidos foram livros sobre DPOC e reabilitação pulmonar disponíveis na em bibliotecas. Além de artigos (escritos nos idiomas português, inglês e espanhol) indexados, livres e bloqueados, porém, disponíveis no sistema virtual das instituições acadêmicas de Salvador, que fossem do tipo ensaio clínico e série de casos e abordassem os efeitos do dos treinos de endurance e força nos sintomas e qualidade de vida de indivíduos com DPOC clinicamente estáveis.

Foram excluídos os estudos constituídos por pacientes que estavam com exacerbação da patologia, aqueles que focaram nas respostas cardiovasculares, que participavam de qualquer outro tratamento adicional ou que utilizassem medicamentos, além daqueles de rotina.

Para a produção dos resultados e confecção do quadro foi empregado o programa Microsoft Office Excel versão 2007.

\section{RESULTADOS}

Após busca em literatura foram selecionados seis artigos publicados nos anos de 2001 a 2010 sobre exercícios de força e de resistência em pacientes com DPOC, sendo quatro no idioma português e dois em inglês. Cinco destes eram ensaios clínicos, sendo quatro deles randomizados $^{(6,7,8,9)}$ e uma é série de caso. ${ }^{(10)}$

O Quadro 1 detalha informações sobre os diferentes trabalhos, mostrando o autor, ano de publicação, revista publicada, grau de comprometimento do sujeito com DPOC, característica da população e os critérios de exclusão determinados por cada estudo. A 
visualização do quadro permite identificar que três estudos avaliaram indivíduos com grau de comprometimento da patologia moderado a grave, ${ }^{(6,8,9)}$ um moderado a muito grave, ${ }^{(7)} \mathrm{um}$ grave e muito grave ${ }^{(11)}$ e um não relata a gravidade, apenas afirma que o diagnóstico era confirmado por um médico. ${ }^{(10)}$ Para classificação destes graus todos utilizaram os critérios GOLD.

Quadro 1 - Exposição dos estudos quanto a revista de publicação, grau de comprometimento da DPOC(gravidade), características da população estudada e critérios de exclusão

\begin{tabular}{|c|c|c|c|c|}
\hline Estudo & Revista & $\begin{array}{l}\text { Grau de } \\
\text { comprometime } \\
\text { nto }\end{array}$ & População & Critérios de exclusão \\
\hline $\begin{array}{l}\text { Ike e col, } \\
2010\end{array}$ & $\begin{array}{l}\text { Fisioterapia em } \\
\text { movimento }\end{array}$ & $\begin{array}{l}\text { DPOC moderada } \\
\text { a muito grave. }\end{array}$ & $\begin{array}{l}12 \text { indivíduos - } 50 \text { a } 82 \\
\text { anos. } \\
\text { Sedentários; não } \\
\text { fumantes ou ex- } \\
\text { fumantes; } 9 \text { homens e } 3 \\
\text { mulheres; IMC < } 30 \\
\mathrm{~kg} / \mathrm{m}^{2}\end{array}$ & $\begin{array}{l}\text { Patologias cardiovasculares, } \\
\text { neurológicas } \\
\text { e/ou osteoarticulares }\end{array}$ \\
\hline $\begin{array}{l}\text { Trevisan } \\
\text { e col, } \\
2010\end{array}$ & $\begin{array}{l}\text { Fisioterapia e } \\
\text { Pesquisa }\end{array}$ & $\begin{array}{l}\text { DPOC } \\
\text { diagnosticada } \\
\text { pelo médico. }\end{array}$ & $\begin{array}{l}9 \text { indivíduos- } 49 \text { a } 76 \\
\text { anos; } \\
\text { Índice de massa } \\
\text { corpórea (IMC): } 52 \text { e } 92 \\
\text { kg; } \\
3 \text { homens e } 6 \text { mulheres. }\end{array}$ & $\begin{array}{l}\text { Fumantes e/ou portadores de } \\
\text { comorbidades(doenças } \\
\text { ortopédicas, reumatológicas, } \\
\text { neurológicas ou cardíacas) }\end{array}$ \\
\hline $\begin{array}{l}\text { Kunikos } \\
\text { hita e } \\
\text { col, } 2006\end{array}$ & $\begin{array}{l}\text { Revista } \\
\text { Brasileira de } \\
\text { Fisioterapia }\end{array}$ & $\begin{array}{l}\text { Moderada a } \\
\text { grave. }\end{array}$ & $\begin{array}{l}25 \text { pacientes; } \\
\text { Homogenia(dados } \\
\text { demográfico, } \\
\text { antropométricos e } \\
\text { espirométricos); } \\
\text { 20homens e } 5 \text { mulheres. }\end{array}$ & $\begin{array}{l}\text { Problemas musculoesqueléticos, } \\
\text { neurológicos, cardiovasculares, } \\
\text { déficit de compreensão; crise } \\
\text { aguda. }\end{array}$ \\
\hline $\begin{array}{l}\text { Wurtem } \\
\text { berger e } \\
\text { col, } 2001\end{array}$ & Pneumologie & $\begin{array}{l}\text { Moderada a } \\
\text { grave }\end{array}$ & $\begin{array}{l}69 \text { indivíduos ( } 44 \\
\text { homens e } 25 \text { mulheres). }\end{array}$ & ----------------- \\
\hline $\begin{array}{l}\text { Marrara } \\
\text { e col, } \\
2008\end{array}$ & $\begin{array}{l}\text { Respiratory } \\
\text { medicine }\end{array}$ & $\begin{array}{l}\text { Moderada a } \\
\text { grave }\end{array}$ & 22 indivíduos & 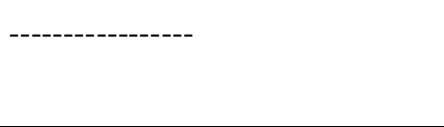 \\
\hline $\begin{array}{l}\text { Ferreira } \\
\text { e col, } \\
2009\end{array}$ & $\begin{array}{l}\text { Jornal Brasileiro } \\
\text { de Pneumologia }\end{array}$ & $\begin{array}{l}\text { Estádios grave } \\
\text { e muito grave. }\end{array}$ & $\begin{array}{l}16 \text { pacientes; ex- } \\
\text { tabagistas,; estáveis } \\
\text { clinicamente; sexo } \\
\text { masculino }(51 \text { a } 80 \text { anos, } \\
\left.\text { IMC } 25 \mathrm{~kg} / \mathrm{m}^{2}\right) \text {; Com } \\
\text { doença cardíaca( } 5- \\
\text { insuficiência valvular, 2- } \\
\text { hipertensão pulmonar e } \\
1 \text { com insuficiência } \\
\text { cardíaca esquerda). }\end{array}$ & ----------------- \\
\hline
\end{tabular}


Quanto à população dos artigos em questão, a quantidade de pessoas variou de nove a 69 indivíduos; a idade variou de 50 a 82, sendo que três não relataram a idade dos indivíduos estudados, referindo apenas a homogeneidade da população. ${ }^{(6,8,11)} \mathrm{O}$ índice de massa corpórea (IMC) foi pontuado em dois estudos $\left(25 \mathrm{~kg} / \mathrm{m}^{2}\right){ }^{(11)}\left(30 \mathrm{~kg} / \mathrm{m}^{2}\right)^{(7)}$ e um trouxe variação de peso entre 52 e $92 \mathrm{Kg}$ (10) $^{(1)}$ foram descritos hábitos de vida da população em dois estudos (sedentários, ex-fumantes, não-fumantes e ex-tabagistas). ${ }^{(7,11)}$ Os estudos possuíam homogeneidade de sujeitos, que apresentavam-se estáveis clinicamente e sem comorbidades (exceto em um trabalho que incluiu indivíduos com doença cardíaca), ${ }^{(1)}$ não estavam no período agudo da patologia e alteraram o tratamento medicamentoso, nem faziam nenhum outro tipo de tratamento adicional. 
Quadro 2 - Artigos selecionados descritos quanto ao tipo de exercício realizado, a frequência, o pré e pós teste e os resultados

\begin{tabular}{|c|c|c|c|c|}
\hline Estudo & Tipo de exercício & Frequência & Resultados & Pré/Pós-teste \\
\hline $\begin{array}{l}\text { Ike e col, } \\
2010 .\end{array}$ & 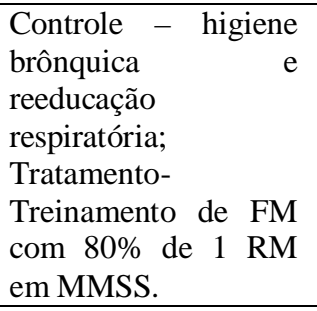 & $\begin{array}{l}3 \text { vezes/ semana }(40 \mathrm{~min}), \text { por } \\
\text { seis semanas }\end{array}$ & $\begin{array}{l}\text { Grupo treinado: Significativo } \\
\text { aumento de Fm, sem interferir } \\
\text { na capacidade funcional. }\end{array}$ & $\begin{array}{llll}\text { Testes de } 1 & \text { RM } & \text { e o } \\
\text { Pegboard and } & \text { Ring } & \text { Test } \\
(\text { PBRT) } & & & \end{array}$ \\
\hline $\begin{array}{l}\text { Trevisan e } \\
\text { col, } 2010 .\end{array}$ & $\begin{array}{l}\text { Fortalecimento da } \\
\text { musculatura } \\
\text { inspiratória, } \\
\text { abdominais } \\
\text { quadríceps }-30 \% \text { de } \\
1 \mathrm{RM} \text {, atingindo ao } \\
\text { final } 60 \% \text {.. }\end{array}$ & Duas vezes/ semana - 2 meses. & $\begin{array}{l}\text { Melhora em todas variáveis: } \\
\text { significativa na PI max } \\
(p<0,05) \text {. } \\
\text { Referiram menor sensação de } \\
\text { cansaço }\end{array}$ & 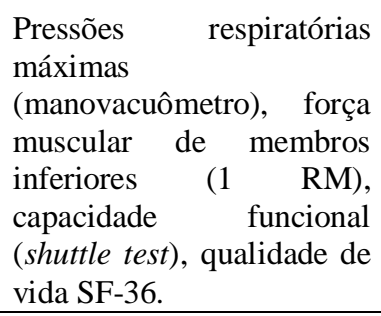 \\
\hline $\begin{array}{l}\text { Kunikoshi } \\
\text { t e col, } \\
2006 .\end{array}$ & $\begin{array}{l}\text { Grupo 1: treino da } \\
\text { musculatura } \\
\text { respiratória com } 30 \% \\
\text { da PI máx obtida a } \\
\text { cada semana } \\
\text { Grupo } 2 \text { : esteira com } \\
70 \% \text { da FC atingida } \\
\text { no teste de exercício } \\
\text { cardiorrespiratório } \\
\text { (TECR); } \\
\text { Grupo 3- ambos } \\
\text { treinamentos. }\end{array}$ & 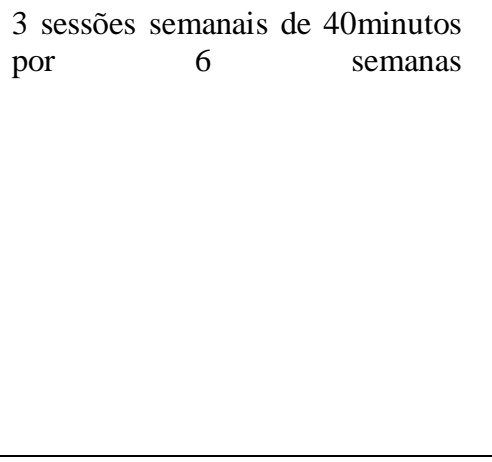 & $\begin{array}{l}\text { Força muscular respiratória } \\
\text { intergrupos não foi } \\
\text { sifinificativa; } \\
\text { Aumento da PImax( No } \\
\text { GRUPO } 1 \text { e 3); Pe max no } \\
\text { G1; TECR: Volume minuto } \\
\text { expirado maior no G2 em } \\
\text { relação ao G1; G2: aumento } \\
\text { significativo no domínio } \\
\text { "capacidade funcional", e o } \\
\text { G3 na pontuação total do } \\
\text { QQV SF36 }\end{array}$ & $\begin{array}{l}\text { Espirometria; força } \\
\text { muscular respiratória } \\
\text { (FMR); Teste de exercício } \\
\text { cardiorrespiratório (TECR) } \\
\text { sintoma-limitado; } \\
\text { qualidade de vida -SF-36. }\end{array}$ \\
\hline $\begin{array}{l}\text { Wurtembe } \\
\text { rger e col, } \\
2001\end{array}$ & $\begin{array}{l}\text { Aeróbico: grupos P1 } \\
\text { e P4; } \\
\text { Força muscular(FM): } \\
\text { P2 e P5; }\end{array}$ & $\begin{array}{l}3 \text { sessões/semanas - } 20 \text { minutos. } \\
\text { Revista Pesquisa em Fisioterapia, Salvador, } \\
\text { http://www.bahiana.edu.br }\end{array}$ & $\begin{array}{l}\text { Dispnéia foi reduzida em } \\
\text { todos os grupos; } \\
\text { Pacientes graves têm maiores } \\
\text { benefícios no de FM isolado }\end{array}$ & $\begin{array}{l}\text { TC6min; tempo para } \\
\text { terminar um conjunto de } \\
\text { atividades diárias(TAF). }\end{array}$ \\
\hline
\end{tabular}




\begin{tabular}{|c|c|c|c|c|}
\hline & $\begin{array}{l}\text { Combinação: } \mathrm{P} 4 \mathrm{e} \\
\text { P6. }\end{array}$ & & $\begin{array}{l}\text { ou associado; os moderados } \\
\text { com o aeróbico. }\end{array}$ & \\
\hline $\begin{array}{l}\text { Marrara e } \\
\text { col, } 2008\end{array}$ & $\begin{array}{l}\text { Grupo1: esteira; } \\
\text { Grupo 2: FM de } \\
\text { MSS; } \\
\text { Grupo 3- Terapia de } \\
\text { higiene brônquica }\end{array}$ & 6 semanas- 3 vezes por semana. & $\begin{array}{l}\text { Dispnéia diminui } \\
\text { significativamente no grupo 1; } \\
\text { Número de escadas diminui } \\
\text { para o grupo } 3 \text { e aumento para } \\
\text { os demais; } \\
\text { A distância da caminhada } \\
\text { aumentou para o 1; Maiores } \\
\text { relatos de dispnéia no 3. }\end{array}$ & $\begin{array}{l}\text { Simuladas AVD'S (PBRT } \\
\text { e levantamento de peso }-5 \\
\text { min; subir escadas e } \\
\text { caminhada na esteira }-6 \\
\text { min). }\end{array}$ \\
\hline $\begin{array}{l}\text { Ferreira e } \\
\text { col, } 2009\end{array}$ & $\begin{array}{l}1 \text { grupo: Caminhada } \\
\text { na esteira - } 80 \% \text { da } \\
\text { frequência cardíaca } \\
\text { máxima. }\end{array}$ & $\begin{array}{l}3 \text { vezes por semana, durante um } \\
\text { período de } 20 \mathrm{~min} \text {, ao longo de } \\
\text { dois meses (total de } 20 \text { sessões). }\end{array}$ & $\begin{array}{l}\text { Melhora significativa na } \\
\text { sensação de dispnéia, } \\
\text { qualidade de vida e } \\
\text { capacidade de exercício. }\end{array}$ & $\begin{array}{l}\text { Dessaturação de oxigênio, } \\
\text { sensação d e dispneia } \\
\text { (escalas de MMRC e de } \\
\text { Borg), qualidade de vida } \\
\text { ( St. George's Respiratory } \\
\text { Questionnaire (SGRQ), } \\
\text { TC6 min. }\end{array}$ \\
\hline $\begin{array}{l}\text { Ike e col, } \\
2010 .\end{array}$ & 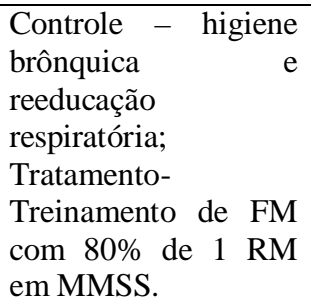 & $\begin{array}{l}3 \text { vezes/ semana(40 min), por } \\
\text { seis semanas }\end{array}$ & $\begin{array}{l}\text { Grupo treinado: Significativo } \\
\text { aumento de Fm, sem interferir } \\
\text { na capacidade funcional. }\end{array}$ & $\begin{array}{llll}\text { Testes de } 1 & \text { RM } & \text { e o } \\
\text { Pegboard and } & \text { Ring } & \text { Test } \\
\text { (PBRT) } & & & \end{array}$ \\
\hline $\begin{array}{l}\text { Trevisan e } \\
\text { col, } 2010 .\end{array}$ & $\begin{array}{l}\text { Fortalecimento da } \\
\text { musculatura } \\
\text { inspiratória, } \\
\text { abdominais } \\
\text { quadríceps }-30 \% \text { de } \\
1 \mathrm{RM}, \text { atingindo ao } \\
\text { final } 60 \% . .\end{array}$ & Duas vezes/ semana - 2 meses. & $\begin{array}{l}\text { Melhora em todas variáveis: } \\
\text { significativa na PI max } \\
(p<0,05) \text {. } \\
\text { Referiram menor sensação de } \\
\text { cansaço }\end{array}$ & 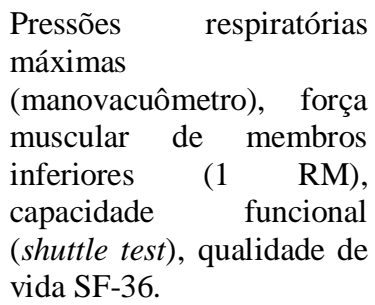 \\
\hline Kunikoshi & Grupo 1: treino da & 3 sessões semanais de 40 minutos & Força muscular respiratória & \\
\hline
\end{tabular}

Revista Pesquisa em Fisioterapia, Salvador, dez. 2012; 2(2): 70-82. 


\begin{tabular}{|c|c|c|c|c|}
\hline $\begin{array}{l}\text { t e col, } \\
2006 .\end{array}$ & $\begin{array}{l}\text { musculatura } \\
\text { respiratória com } 30 \% \\
\text { da PI máx obtida a } \\
\text { cada semana } \\
\text { Grupo } 2 \text { : esteira com } \\
70 \% \text { da FC atingida } \\
\text { no teste de exercício } \\
\text { cardiorrespiratório } \\
\text { (TECR); } \\
\text { Grupo 3- ambos } \\
\text { treinamentos. }\end{array}$ & semanas & $\begin{array}{l}\text { intergrupos não } \\
\text { sifinificativa; } \\
\text { Aumento da PImax ( No } \\
\text { GRUPO 1 e 3); Pe max no } \\
\text { G1; TECR: Volume minuto } \\
\text { expirado maior no G2 em } \\
\text { relação ao G1; G2: aumento } \\
\text { significativo no domínio } \\
\text { "capacidade funcional", e o } \\
\text { G3 na pontuação total do } \\
\text { QQV SF36 }\end{array}$ & 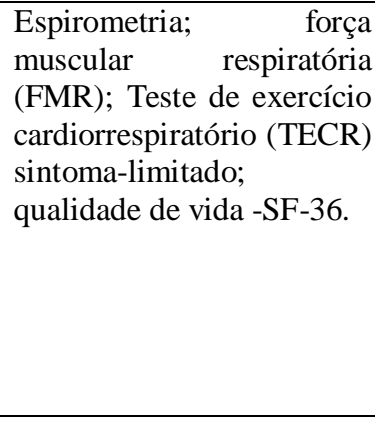 \\
\hline $\begin{array}{l}\text { Wurtembe } \\
\text { rger e col, } \\
2001\end{array}$ & $\begin{array}{l}\text { Aeróbico: grupos P1 } \\
\text { e P4; } \\
\text { Força muscular(FM): } \\
\text { P2 e P5; } \\
\text { Combinação: P4 e } \\
\text { P6. }\end{array}$ & 3 sessões/semanas - 20 minutos. & $\begin{array}{l}\text { Dispnéia foi reduzida em } \\
\text { todos os grupos; } \\
\text { Pacientes graves têm maiores } \\
\text { benefícios no de FM isolado } \\
\text { ou associado; os moderados } \\
\text { com o aeróbico. }\end{array}$ & $\begin{array}{l}\text { TC6min; tempo para } \\
\text { terminar um conjunto de } \\
\text { atividades diárias(TAF). }\end{array}$ \\
\hline $\begin{array}{l}\text { Marrara e } \\
\text { col, } 2008\end{array}$ & $\begin{array}{l}\text { Grupo1: esteira; } \\
\text { Grupo 2: FM de } \\
\text { MSS; } \\
\text { Grupo 3- Terapia de } \\
\text { higiene brônquica }\end{array}$ & 6 semanas- 3 vezes por semana. & $\begin{array}{l}\text { Dispnéia } \\
\text { significativamente no grupo 1; } \\
\text { Número de escadas diminui } \\
\text { para o grupo } 3 \text { e aumento para } \\
\text { os demais; } \\
\text { A distância da caminhada } \\
\text { aumentou para o 1; Maiores } \\
\text { relatos de dispnéia no 3. }\end{array}$ & $\begin{array}{l}\text { Simuladas AVD'S (PBRT } \\
\text { e levantamento de peso - } 5 \\
\text { min; subir escadas e } \\
\text { caminhada na esteira }-6 \\
\text { min). }\end{array}$ \\
\hline $\begin{array}{l}\text { Ferreira e } \\
\text { col, } 2009\end{array}$ & $\begin{array}{l}1 \text { grupo: Caminhada } \\
\text { na esteira - } 80 \% \text { da } \\
\text { frequência cardíaca } \\
\text { máxima. }\end{array}$ & $\begin{array}{l}3 \text { vezes por semana, durante um } \\
\text { período de } 20 \mathrm{~min} \text {, ao longo de } \\
\text { dois meses (total de } 20 \text { sessões). }\end{array}$ & $\begin{array}{l}\text { Melhora significativa na } \\
\text { sensação de dispnéia, } \\
\text { qualidade de vida e } \\
\text { capacidade de exercício. }\end{array}$ & $\begin{array}{l}\text { Dessaturação de oxigênio, } \\
\text { sensação d e dispneia } \\
\text { (escalas de MMRC e de } \\
\text { Borg), qualidade de vida } \\
\text { ( St. George's Respiratory } \\
\text { Questionnaire (SGRQ), } \\
\text { TC6 min. }\end{array}$ \\
\hline
\end{tabular}


No Quadro 2 é possível verificar a frequência, tipo de treino, os resultados do estudo e o pré e pós teste realizado em cada um. Nos artigos observa-se que a frequência dos atendimentos foi de duas a três sessões por semana com tempo de 20 a 40 minutos em cada um dos encontros, realizados durante 6 a 12 semanas. $\mathrm{O}$ tipo de tratamento variou entre treinamento de força e treinamento respiratório; ${ }^{(7,10)}$ grupo com exercícios aeróbicos e ambos; ${ }^{(6)}$ grupo aeróbico, grupo de fortalecimento e grupo com as duas modalidades; ${ }^{(8)}$ treinamento na esteira, grupo de fortalecimento de membros superiores e grupo utilizando terapia de higiene brônquica; ${ }^{(9)}$ sessões com caminhadas na esteira. ${ }^{(11)}$

Foi relatado em quatro estudos a redução da sensação de cansaço (dispnéia) ${ }^{(8,9,10,11)}$ sendo que um não citou esta variável ${ }^{(7)}$ e outro citou aumento de PiMáx (pressão inspiratória) e PEmáx (pressão expiratória máxima); ${ }^{(6)}$ a tolerância ao exercício aumentou em cinco dos trabalhos; $;^{(6,8,9,10,11)}$ a qualidade de vida melhorou em três ${ }^{(6,10,11)}$ artigos e não foi avaliada nos demais.

Para avaliação das variáveis foram mais frequentes os testes de 1 RM (uma resistência máxima), o questionário de qualidade de vida SF-36, o teste de caminhada de seis minutos (TC6 min); e para avaliar a dispnéia foi utilizada a escala de Borg e de MRC, ${ }^{(11)}$ mas a maioria dos trabalhos apenas relatou ter havido uma diminuição da sensação de cansaço referida pelo paciente antes e após o tratamento, porém, sem mensurá-la. ${ }^{(8,9,10)}$

\section{DISCUSSÃO}

Diante dos resultados encontrados nesta pesquisa, pode-se entender, que o treino de endurance e força têm influencia positiva nos sintomas de dispnéia (apesar de poucos estudos utilizarem escalas direcionadas para a sua mensuração), tolerância ao esforço e na qualidade de vida de sujeitos com idades entre 50 a 82 anos (principalmente naqueles que não tenham co morbidades). Sendo estes achados independentes do estadiamento da patologia.

Constatou-se também que existe na literatura um consenso de frequência de treinamento de 2 a 3 vezes na semana, sendo mais frequente 3 sessões, e que a duração destas sessões varia entre 20 a 40 minutos, não divergindo os benefícios finais se desenvolvidos programas por 6 a 12 semanas. Porém, não são tão discutidos protocolos de séries, repetições e cargas, nem de percentual de 1 RM e FC de treino, mesmo sendo encontrados treinos de força com 60 a $80 \%$ de $1 \mathrm{RM}^{(7,10)}$ e treinos aeróbicos com $70 \%$ a $80 \%$ da freqüência cardíaca (FC) máxima ${ }^{(6,11)}$ com melhoras nas variáveis discutidas. 
Uma revisão publicada em 2004 sobre recondicionamento muscular nestes pacientes relata que o exercício aeróbico e o treino de força são fundamentais no incremento da capacidade física e qualidade de vida principalmente em indivíduos que apresentam formas moderadas ou graves da DPOC como detectado neste estudo. Ainda nesta revisão, são citados artigos que compararam o treinamento de membros inferiores no cicloergômetro com um grupo controle não condicionado que obteve melhora significativa no TC6 min, na VO2 pico, no tempo de endurance com carga constante em cilcoergômetro, na PI máx e na sensação de dispnéia no grupo treinado; sugerindo então, melhora global no indivíduo mesmo na ausência de outros componentes importantes no processo de reabilitação pulmonar, como treinamento respiratório, otimização da clearance de vias aéreas, dentre outros. ${ }^{(12)}$

A revisão traz um estudo que associa o treino dos músculos respiratórios com $40 \%$ da PI máx a exercícios aeróbicos duas vezes por semana identificando a melhora no TC6 min e, consequentemente, melhora na capacidade funcional e tolerância ao esforço somente no grupo aeróbico, apesar de não relatar a duração da terapia; discute também, que exercícios feitos entre $60 \%$ a $80 \%$ da carga máxima de trabalho determinam maiores benefícios. ${ }^{(12)}$ No presente trabalho foi visto que num estudo que dividiu três grupos e aplicou treino de musculatura respiratória com 30\% da PI máx em um grupo, esteira com $70 \%$ da FC máxima no segundo grupo e o terceiro grupo associando ambos os treinamentos, verificou melhora significativa no domínio capacidade funcional no grupo 2 e uma maior pontuação total no SF36 no grupo $3 .^{(6)}$

Quanto ao treino de força, a revisão trouxe dois estudos com pacientes graves, porém sem determinar qual critério foi utilizado para descriminar esta categoria. No primeiro foi feito treino progredindo ao longo do tratamento de $50 \%$ para $85 \%$ de 1 RM, onde não foi percebido aumento significativo no TC6min, mas melhora na qualidade de vida quando comparado com o grupo controle. No outro, não foram expostas as características do treino, mas foi identificado aumento no TC6 min e na qualidade de vida. ${ }^{(12)}$ No presente estudo, viuse que sujeitos graves têm maiores benefícios nos treinos de força muscular isolado ou associado ao treinamento aeróbico e que a união dos treinamentos demonstraram uma melhora na qualidade de vida quando comparado com o treinamento de aeróbico isolado ou somente o treinamento da musculatura respiratória. ${ }^{(8)}$

Em relação à associação dos treinos, a revisão sugere que os achados corroboram para uma melhora da força muscular e da capacidade para realizar exercícios nos dois regimes de 
treinamento. Entretanto, o treino de força isolado demonstrou maior associação com melhora da resistência muscular de quadríceps, da resistência aeróbica no teste com carga constante na esteira e da qualidade de vida nos pacientes. ${ }^{(12)}$ Já no estudo em questão foi identificado que se associado nas sessões treinamento da musculatura inspiratória, abdominais e quadríceps com evolução de $30 \%$ a $60 \%$ de 1 RM duas vezes por semana durante dois meses obtêm-se crescimento significativo nos valores de PI máxima, menor sensação de esforço percebido no shuttler test, além de aumento na pontuação do questionário de qualidade de vida. ${ }^{(10)}$

Quanto à qualidade de vida, o seu maior número de pontos total não se deve há índices espirométricos, mas sim a $\mathrm{PaCO} 2$ e a melhora da dispnéia, sendo este o principal atributo clínico capaz de predizer esta variável. ${ }^{(13)}$ Mas, obviamente, qualidade de vida é um conceito muito amplo e subjetivo que não se restringe a fatores mensuráveis e sim a maneira de encarar a vida, do significado dado a mesma, envolve inúmeras questões próprias de cada sujeito não podendo definir uma hipótese fechada, sendo até os benefícios de socialização (presente em grupos de treinamento) capazes de interferir nesta modificação.

$\mathrm{Na}$ musculatura periférica, evidências comprovam que existe diminuição na concentração de enzimas oxidativas, redução de massa muscular e força, além do menor metabolismo de fosfocreatina em indivíduos com DPOC, sendo as perdas mais acentuadas em decorrência da maior inatividade (o que explica o menor comprometimento dos membros superiores por realizarem uma quantidade de atividades diárias maior). $\mathrm{O}$ treino resistido parece aumentar a força muscular e o metabolismo da fosfocreatina; e o treino aeróbico aumentar VO2 máximo e a concentração de enzimas oxidativas, além de dar um maior condicionamento cardiovascular, o que desencadeia uma melhora da tolerância ao exercício e menor dispnéia. ${ }^{(14)}$

Além dos fatores relacionados a uma menor efetividade do sistema musculoesquelético, sugere-se que a baixa reserva ventilatória também possa estar associada à sensação de dispnéia. ${ }^{(14)}$ Os treinamentos de endurance e força parecem interferir neste sintoma ao otimizarem a função muscular. Sendo sugerido que o treino de força em membros inferiores influencia positivamente a função pulmonar, pois melhora o VEF1, a capacidade vital forçada e a relação $\mathrm{VEF}_{1} / \mathrm{CVF}{ }^{(15)}$

Em suma, as condutas realizadas para os pacientes com DPOC devem englobar não só exercícios para a musculatura respiratória e uso de medicamentos, mas outras que envolvam treinos aeróbicos e anaeróbicos de todo o corpo, enfatizando os músculos da deambulação, 
músculos dos membros superiores, latíssimo do dorso, peitoral maior e abdominais durante um mínimo de seis semanas de treino por 2 a 3 vezes por semana, ${ }^{(16)}$ sendo necessário maiores estudos que protocolem número de repetições e séries e que avaliem diretamente a função pulmonar.

\section{REFERÊNCIAS}

1. Sociedade Brasileira de Pneumologia e Tisiologia. Consenso Brasileiro sobre Doença Pulmonar Obstrutiva crônica - DPOC. J. bras. pneumol. 2004; 30(Suppl 5): 1-5/33-34.

2.Tarantino AB. Doenças pulmonares. 4ª ed. Rio de Janeiro: Guanabara Koogan; 1997.

3. OMS - Organização Mundial da Saúde. Who report on the global tobacco epidemic. São Paulo, 2008 [acesso em 20 out. 2011]. Disponível em: <http://www.who.int/tobacco/mpower/mpower_report_full_2008.pdf>

4. Langer D et al. Guia para prática clínica: Fisioterapia em pacientes com Doença Pulmonar Obstrutiva Crônica (DPOC). Rev. bras. fisioter. 2009; 13(3): 183-204.

5. Godoy R, et al. Repercussões tardias de um programa de reabilitação pulmonar sobre os índices de ansiedade, depressão, qualidade de vida e desempenho físico em portadores de DPOC. J. bras. pneumol. 2009; 35(2): 129-136.

6. Kunikoshita LN et al. Efeitos de três programas de fisioterapia respiratória (PFR) em portadores de DPOC. Rev. bras. fisioter. 2006; 10(4): 449-455.

7. Ike D et al. Efeitos do exercício resistido de membros superiores na força muscular periférica e na capacidade funcional do paciente com DPOC. Fisioter. mov. 2010; 23(3): 429437.

8. Wurtemberger G, et al. Functional effects of different training in patients with COPD. Pneumologie. 2001; 55(12): 553-62.

9. Marrara KT et al. Different physical therapy interventions on daily physical activities in chronic obstructive pulmonary disease. Respir. med. 2008; 102(4): 505-11.

10. Trevisan $M$ et al. Influência do treinamento da musculatura respiratória e de membros inferiores no desempenho funcional de indivíduos com DPOC. Fisioter. pesqui. 2010; 17(3): 209-213.

11. Ferreira F, Guimaraes M, Taveiran N. Reabilitação respiratória na DPOC: do treinamento de exercício para a "vida real". J. bras. pneumol. 2009; 35(11): 1112-1115.

Revista Pesquisa em Fisioterapia, Salvador, dez. 2012; 2(2): 121-133. 
12. Dourado V et al. Recondicionamento muscular na DPOC: principais intervenções e novas tendências. Rev. bras. educ. fís. esp. 2004; 10(4): 331-334.

13. Dourado V et al. Fatores associados à diferença clinicamente significativa da qualidade de vida relacionada à saúde após condicionamento físico em pacientes com DPOC. J. bras. pneumol. 2009; 35(9): 846-853.

14. Silva E, et al. Treinamento de força para pacientes com doença pulmonar obstrutiva crônica. Rev. bras. educ. fís. esp. 2008; 14(3): 231-238.

15. Regueiro E, et al. Análise da demanda metabólica e ventilatória durante a execução de atividades de vida diária em indivíduos com doença pulmonar obstrutiva crônica. Revista Latino-americana de Enfermagem. 2006; 14(1): 41-7.

16. Dourado V, et al. Effect of three exercise programs on patients with chronic obstructive pulmonary disease. Braz. j. med. biol. res. 2009; 42(3): 263-271. 